\title{
A multiport isolated DC-DC converter
}

Y. K. Tran and D. Dujic

This material is posted here with permission of the IEEE. Such permission of the IEEE does not in any way imply IEEE endorsement of any of EPFL's products or services. Internal or personal use of this material is permitted. However, permission to reprint / republish this material for advertising or promotional purposes or for creating new collective works for resale or redistribution must be obtained from the IEEE by writing to pubs-permissions@ieee. org. By choosing to view this document, you agree to all provisions of the copyright laws protecting it. 


\title{
A Multiport Isolated DC-DC Converter
}

\author{
Yan-Kim Tran, Drazen Dujic \\ Power Electronics Laboratory - PEL \\ École Polytechnique Fédérale de Lausanne - EPFL \\ Lausanne CH-1015, Switzerland \\ yan-kim.tran@epfl.ch,drazen.dujic@epfl.ch
}

\begin{abstract}
This paper presents a multi-port isolated DC-DC converter for DC applications. A three-port structure is presented, characterized with full bidirectional power flow and simple control. Galvanic isolation is achieved by means of a multi-winding medium frequency transformer which is a part of a resonant LLC converter. To provide controllable power exchange with external DC ports, two out of three ports are equipped with additional bidirectional buck/boost stages. They serve to provide active power flow control, while the inner resonant stage provides galvanic isolation, operates in the open-loop and adapts its mode of operation based on the actual power flow. Both switched mode and rea-time hardware-in-the-loop simulations, with control algorithm deployed on the digital signal processor, are used to verify and demonstrate various operational modes.
\end{abstract}

\section{INTRODUCTION}

Most of the newly installed energy sources (e.g. PV) or storage technologies (e.g. batteries) are DC by nature, resulting in an increased interest in the development of small or large scale DC grids. Flexibility and simplicity of interfacing power electronics as well as suitable protection technologies are the key to enable new grid architectures. Considering energy production, consumption and storage, multi-port power electronic converters are viable solution to bring together all three aspects and meet demands of micro-grids, while being fully flexible for the integration of different technologies on each side.

So far, only a few investigations related to high power multiport converters have been reported. The work of [1] deals with converters based on interconnection through a common DC bus. Despite some advantages due to the simplicity of the control, the voltage range is limited and defined by choice of the bus voltage. This limitation can be overcome by means of electromagnetic coupling through medium or high frequency transformers. In [2],[3] and [4] authors have presented solutions derived from the Dual Active Bridge (DAB) or Dual Half Bridge (DHB) with inclusion of additional ports. Limited soft switching operating range of $\mathrm{DAB}$, resulted in considerations towards the resonant converters thanks to their advantages in reduced switching losses. A multi-port LLC converter has been presented in [5] and consists of a three winding medium frequency transformer (MFT) with distributed resonant capacitors tuned to multiple leakage inductors, in order to create resonant tank. The authors have presented configuration where one port act as a source, while two other ports are acting as sink (load). Experiments from a low power setup (300W), demonstrate good load regulation but gave no consideration to different operating modes. On the other hand, work of [6] shows a similar topology where two ports are configured as sources and are equipped with resonant capacitors, while the third port has only a rectifier as it is behaving as the load. Authors have demonstrated controllable power flow and load sharing between sources, using phase shift control. While, several works have been dealing already with LLC or multiport converters, the focus has been mainly on a low power rated converters and unidirectional power flow.

This paper is an extension of the previous work [7] and it presents a novel multi-port topology and its operation in section II. Design considerations and sizing of main components are presented in section III, while the control scheme is provided in section IV. PLECS simulations as well as hardwarein-the-Loop simulations, with control algorithm deployed on the digital signal processor (DSP) are given in section V. A summary and conclusion are provided in section VI.

\section{TOPOlOgy AND Operating Modes}

The topology considered in this paper is based on a three winding medium frequency transformer as shown in Fig. 1. It is fully bidirectional, even with one of the three ports deactivated. The converter basic stage is composed of a multi-port LLC resonant circuit equipped with two additional buck/boost stages. The ports may have different power ratings $\left(P_{1} \neq P_{2} \neq\right.$ $\left.P_{3}\right)$ and arbitrary output voltages $\left(V_{D C_{1}} \neq V_{D C_{2}} \neq V_{D C_{3}}\right)$.

\section{A. Multiport LLC Resonant Stage}

The inner part is made by a three winding MFT with turn ratio $n_{i}$ according to the desired output voltages and equipped with three half bridges. In order to achieve high efficiency, a resonant LLC topology is used and operated in the open-loop mode below resonant frequency [8]. Since the full bi-directionality of the converter implies certain symmetry requirements, the resonant tank is not located on a single port, like in [5], but is split between all three ports. Each resonant tank is composed of a capacitor $C_{i}$ and an inductor $L_{i}$ which is the combination of the stray inductor of the MFT and, if needed, an additional inductor. The design of the resonant tank requires proper sizing of all distributed $L_{i}$ and $C_{i}$ in a way that guaranties soft switching conditions for any operating point within the power specifications. The resonant part is intended to be operated as a DC transformer without any complex control scheme. It is only controlled by activating or deactivating PWM on $S_{1,2,3}$, depending on the power flow direction. Only the ports that are providing power 


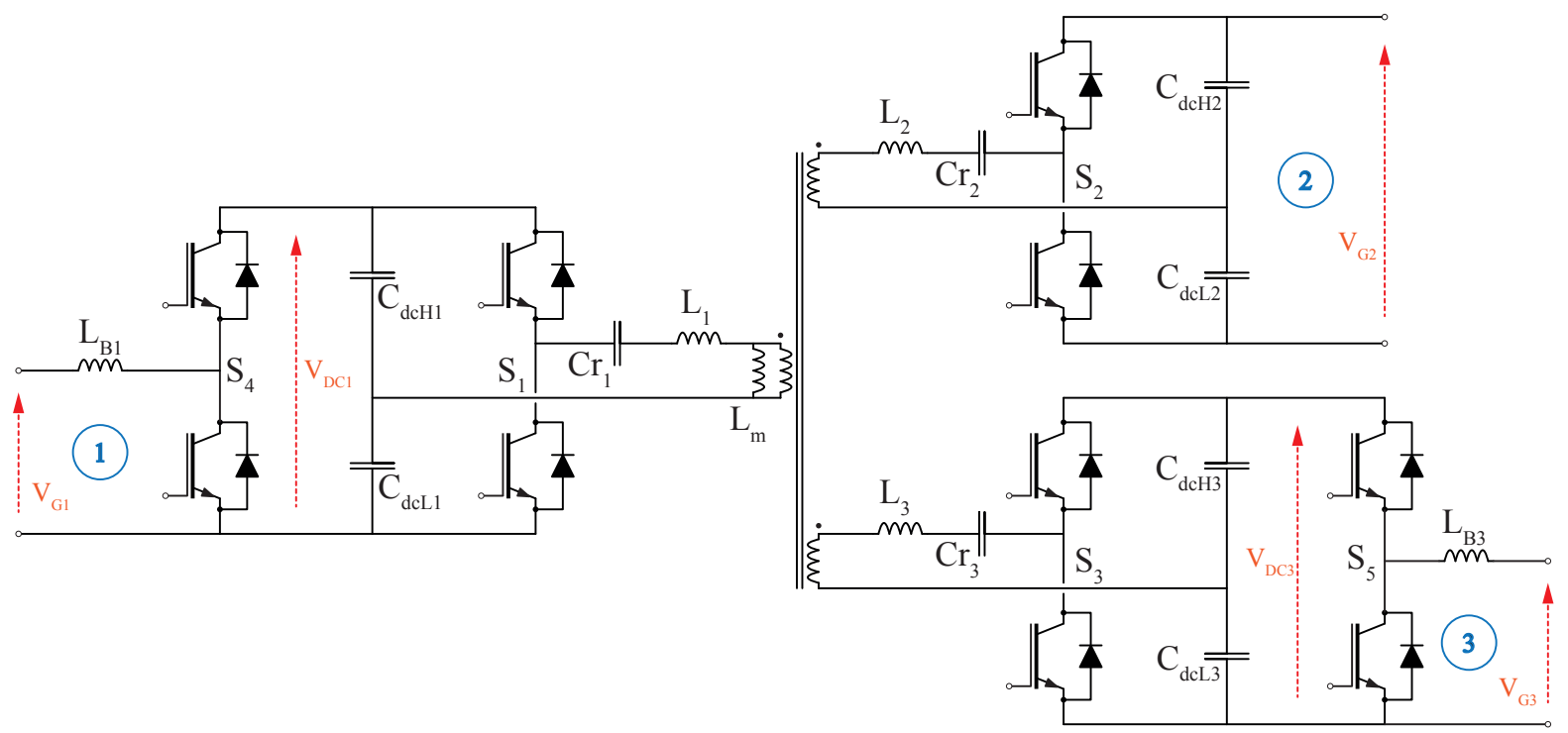

Fig. 1. Topology of a Multiport Isolated DC-DC Converter

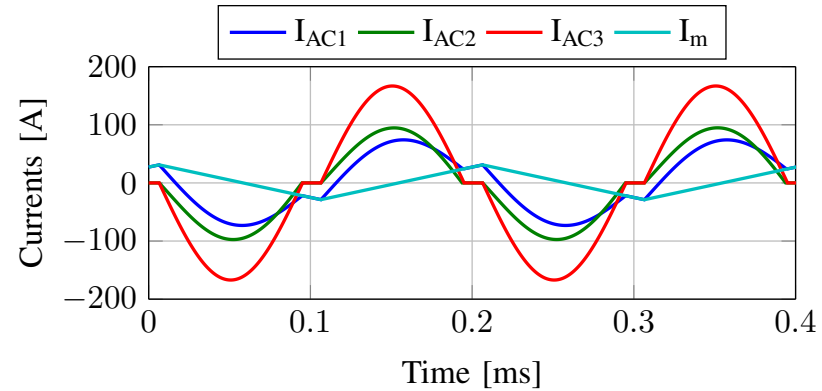

Fig. 2. Source port: $1\left(P_{1}=90 k W\right)$; Sink ports: $2\left(P_{2}=-50 k W\right)$ and 3 $\left(P_{3}=-40 k W\right)$

are actively switched. Load ports are turned off and their freewheeling diodes are used as passive rectifiers (voltage doubler configuration as shown in Fig. 1). When a port is active, its high and low side switches are operated with opposite polarity at a fixed frequency $f_{s w}$ and a constant duty cycle of approximately $50 \%$ (considering dead-time). Typical current waveforms in such a structure are shown in Fig. 2 and Fig. 3.

\section{B. Buck/Boost Stages}

Since no active control is applied on the inner resonant stage, the power flow must be regulated by two additional stages $\left(S_{4}\right.$ and $\left.S_{5}\right)$ on the ports 1 and 3 . These stages are based on a bidirectional buck/boost converter and thus can be operated either as boost or as buck depending on the actual mode of operation, as it will be discussed shortly.

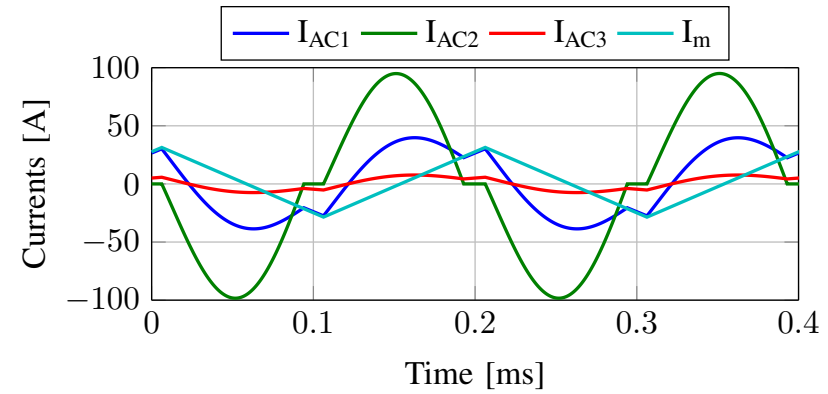

Fig. 3. Source ports: $1\left(P_{1}=50 \mathrm{~kW}\right)$ and $3\left(P_{3}=10 \mathrm{~kW}\right)$; Sink port: 2 $\left(P_{2}=-60 k W\right)$

\section{Operating Modes}

There are several operating modes of this converter that influence the overall control scheme, as summarized in Table I. The resonant stage modes, related to switching cells $S_{1}, S_{2}$ and $S_{3}$, simply describe if the respective switching cells are active (PWM on) or passive (PWM off). However, the operation of $S_{4}$ and $S_{5}$ define if the stage is used as boost or buck converter. In this paper, ports 1 and 2 are the main ports rated for the full power of the converter and in charge of the power flow. Bidirectional operation implies that power flow from the port 1 to the port 2 (modes $\mathrm{A} 1, \mathrm{~B} 1$ and $\mathrm{C} 1$ ) or vice versa (modes A2, B2 and C2). In both cases, the voltage on port 2 is directly controlled through port 1 by the buck/boost stage $S_{4}$. Port 3 is considered as an additional port that can source (mode B1 and B2) or sink (mode A1 and A2) power with reduced ratings, regardless of the power direction between the ports 1 and 2. Its buck/boost stage $S_{5}$, regulates the current $I_{b 3}$, compensating 
for voltage variations due to cross-coupling with ports 1 and 2. In the modes $\mathrm{C} 1$ and $\mathrm{C} 2$, no energy is exchanged with the port 3 and $S_{5}$ is deactivated.

Previous description assumes that multiport converter controls its voltage on port 2 , however in case of presence of additional converter, (e.g. AC grid connected inverter), overall role of different ports may change, as it is usually the grid side converter that controls its DC link voltage (e.g. port 2 voltage).

TABLE I

OPERATING MODES

\begin{tabular}{|l|l|l|l|l|l|}
\hline Mode & $S_{1}$ & $S_{2}$ & $S_{3}$ & $S_{4}$ & $S_{5}$ \\
\hline A1 & Active & Passive & Passive & Boost & Buck \\
\hline A2 & Passive & Active & Passive & Buck & Buck \\
\hline B1 & Active & Passive & Active & Boost & Boost \\
\hline B2 & Passive & Active & Active & Buck & Boost \\
\hline C1 & Active & Passive & Passive & Boost & Off \\
\hline C2 & Passive & Active & Passive & Buck & Off \\
\hline
\end{tabular}

\section{DESIGN METHODOLOGY}

For this case study, power and voltage ratings are chosen somewhat arbitrarily and are summarized in Table II.

TABLE II

POWER AND VOLTAGE RATINGS USED FOR THE CASE STUDY

\begin{tabular}{|l|l|l|l|}
\hline Port & $P_{i}$ & $V_{D C_{i}}$ & $V_{G_{i}}$ \\
\hline 1 & $125 \mathrm{~kW}$ & $3600 \mathrm{~V}$ & $3000 \mathrm{~V}$ \\
\hline 2 & $125 \mathrm{~kW}$ & $1500 \mathrm{~V}$ & $1500 \mathrm{~V}$ \\
\hline 3 & $25 \mathrm{~kW}$ & $750 \mathrm{~V}$ & $400 \mathrm{~V}$ \\
\hline
\end{tabular}

\section{A. LLC Tank Design}

The design of the resonant part is based on a methodology presented in [7]. The aim is to determine the resonant parameters in such a way that ensures the ZVS of the primary switches (active port), soft commutation of rectifier diodes (passive port) and minimize the amount of circulating energy (conduction losses). For a given ratio $f_{n}$, of the switching and the resonant frequency and for a given operating point represented by an $\mathrm{AC}$ load $R_{A C_{i}}$, the maximum value of $L_{r_{i}}$ required to stay in the inductive region is given by:

$$
L_{\max _{i}}=\frac{f_{n} R_{A C_{i}} \pi^{2}}{8 \sqrt{2} \omega_{0}}
$$

The quality factor $Q_{o p t_{i}}$ is defined as the ratio between the load and the tank impedance, under the maximum load conditions, for which the tank RMS current is the smallest. For the operating point given by $R_{A C_{i}}$, the resonant inductor which provides the optimal quality factor is defined, according to [9] and [10], as:

$$
\begin{aligned}
L_{o p t_{i}} & =\frac{R_{A C_{i}}^{2}}{\omega_{0}^{2} L_{m_{i}}} \\
Q_{o p t_{i}} & =\frac{R_{A C_{i}}}{\omega_{0} L_{m_{i}}}
\end{aligned}
$$

We can then obtain the optimal $Q$ factor for each port. The distributed resonant inductors are calculated from the equations linking the three power quality factors to the three resonant inductors:

$$
\begin{aligned}
& L_{i} \leq Q_{\text {opt }_{i}} R_{A C_{i}}-\left(\frac{1}{\left(n_{i} / n_{j}\right)^{2} L_{j}}+\frac{1}{\left(n_{i} / n_{k}\right)^{2} L_{k}}\right)^{-1} \\
& C_{i}=\frac{1}{L_{i}\left(2 \pi f_{\text {res }}\right)^{2}} ; i \neq j \neq k
\end{aligned}
$$

Resulting parameters for the $L_{i}$ and the $C_{i}$ are shown in Table III.

In order to benefit from the ZVS, the magnetizing inductor has to be sized accordingly. Its value will define the turn-off current of the active ports and thus turn-off switching losses. Since this turn-off current corresponds to the negative turnon current of the free-wheeling diode of the complementary switch, its value cannot be selected too low. To preserve ZVS, the resonant tank current should not change polarity within the dead-time. The duration of diode conduction can be approximated by division of the turn-off current by the slope of the resonant tank current at this point. To maintain ZVS within the dead-time $T_{d t}, L_{m}$ must satisfy:

$$
\begin{aligned}
I_{o f f_{m i n}} & \approx T_{d t} I_{D C_{n}} \frac{\pi}{2} \\
L_{m_{i}} & \leq \frac{V_{D C_{i}} T_{s w}}{8 I_{o f f_{m i n}}}
\end{aligned}
$$

Considering that the quality factor $Q$ is linked to the magnetizing inductor through (3), it has to be chosen such to keep the optimal $L_{\text {opt }}$, smaller than $L_{\text {max }}$ :

$$
L_{m_{i}} \geq \frac{R_{A C_{i}} 8 \sqrt{2}}{\omega_{0} f_{n} \pi^{2}}
$$

The magnetizing inductors seen from each port, $L_{m_{i}}$ must fulfill the condition of (7) and (8) for all three ports:

$$
L_{m_{1}}=L_{m} \quad L_{m_{2}}=L_{m} \frac{n_{2}^{2}}{n_{1}^{2}} \quad L_{m_{3}}=L_{m} \frac{n_{3}^{2}}{n_{1}^{2}}
$$

Resulting parameters of the distributed resonant tank, for the ratings used in this paper, are summarized in Table III.

\section{B. DC Link Capacitors Sizing}

DC link capacitors are sized according to voltage ripple specifications and resulting values are shown in Table IV.

$$
\begin{aligned}
C_{D C_{i}} & =\frac{V_{D C_{i}} T_{s w}}{2 R_{d c} \Delta V_{i}} \\
C_{d c H_{i}} & =C_{d c L_{i}}=2 C_{D C_{i}}
\end{aligned}
$$

Since DC capacitors are in series with the resonant tank, resonant capacitors are adjusted to match the value calculated in (5).

$$
C_{r_{i}}=\frac{C_{i} 4 C_{D C_{i}}}{4 C_{D C_{i}}-C_{i}}
$$


TABLE III

DISTRIBUTED RESONANT TANK PARAMETERS

\begin{tabular}{|l|l|l|l|}
\hline$L_{m}$ & $10.0 \mathrm{mH}$ & $f_{\text {sw }}$ llc & $5 \mathrm{kHz}$ \\
\hline$L_{1}$ & $13.7 \mu \mathrm{H}$ & $C_{1}$ & $66.6 \mu \mathrm{F}$ \\
\hline$L_{2}$ & $2.6 \mu \mathrm{H}$ & $C_{2}$ & $354.1 \mu \mathrm{F}$ \\
\hline$L_{3}$ & $29.8 \mu \mathrm{H}$ & $C_{3}$ & $30.7 \mu \mathrm{F}$ \\
\hline
\end{tabular}

TABLE IV

DC LINK CAPACITORS FOR EACH PORT

\begin{tabular}{|l|l|l|l|l|}
\hline Port $_{i}$ & $\Delta V_{i} / V_{d c_{i}}$ & $\Delta V_{i}$ & $C_{D C_{i}}$ & $C_{r_{i}}$ \\
\hline 1 & 0.01 & $36 \mathrm{~V}$ & $96.5 \mu F$ & $80.5 \mu F$ \\
\hline 2 & 0.01 & $15 \mathrm{~V}$ & $555.6 \mu F$ & $421.2 \mu F$ \\
\hline 3 & 0.01 & $7.5 \mathrm{~V}$ & $444.5 \mu F$ & $31.23 \mu F$ \\
\hline
\end{tabular}

\section{Buck/Boost Stage Design}

The switching frequencies for the two buck/boost stages are chosen in such a way that switching frequency of the port 1 is lower than the LLC switching frequency, while the switching frequency of the port 3 is higher. It should be noted that present values are not result of any optimization, since actual hardware design is not the main scope of this paper. Chosen values are shown in Table V. The buck/boost inductors are sized according to the ripple specifications.

$$
L_{B_{i}}=\frac{V_{G_{i}} T_{s w_{\text {boosti }}}}{2 \Delta I_{b_{i}}}
$$

TABLE V

BUCK/BOOST STAGES PARAMETERS

\begin{tabular}{|l|l|l|l|l|l|}
\hline$F_{B_{1}}$ & $2.5 \mathrm{kHz}$ & $\Delta I b 1$ & $4 \mathrm{~A}$ & $L_{B_{1}}$ & $40 \mathrm{mH}$ \\
\hline$F_{B_{3}}$ & $10 \mathrm{kHz}$ & $\Delta I b 3$ & $4 \mathrm{~A}$ & $L_{B_{3}}$ & $5 \mathrm{mH}$ \\
\hline
\end{tabular}

\section{Control System Design}

Simplified control scheme is presented here, considering different ratings for the ports. The output voltage of the port 2 is regulated from the port 1 buck/boost $S_{4}$ stage while the power through port 3 is locally regulated by its own buck/boost stage $S_{5}$. In this way a large degree of freedom exists for integration of different energy storage technologies on port 3 .

\section{A. Plant model}

Considering that the resonant stage is operated in an openloop with a fixed switching frequency, its DC transfer function (gain) is considered constant and close to unity. For that reason it is neglected in the analysis and the DC link capacitors can be considered in parallel, taking into account the turn ratio of the transformer. Thus the circuit can be modeled as shown in Fig. 4. The voltage of the port 2, proportional to the voltage $V_{C_{D C}}^{\prime}$ on $C_{D C}^{\prime}(14)$, has to be regulated and the control input is the duty-cycle $D_{1}$ of the buck/boost stage $S_{4}$. The perturbations to this circuit are the voltage on the buck/boost $S_{4}$ input $V_{G 1}$ and the currents in ports 2 and $3, I_{2}^{\prime}(15)$ and $I_{3}^{\prime}(16)$, respectively (all referred to port 1 side). The buck/boost stage $S_{5}$ current $\left(I_{b 3}\right)$ has to be regulated and the control input is its dutycycle $D_{3}$. The perturbation to this part is the voltage on $C_{D C}$
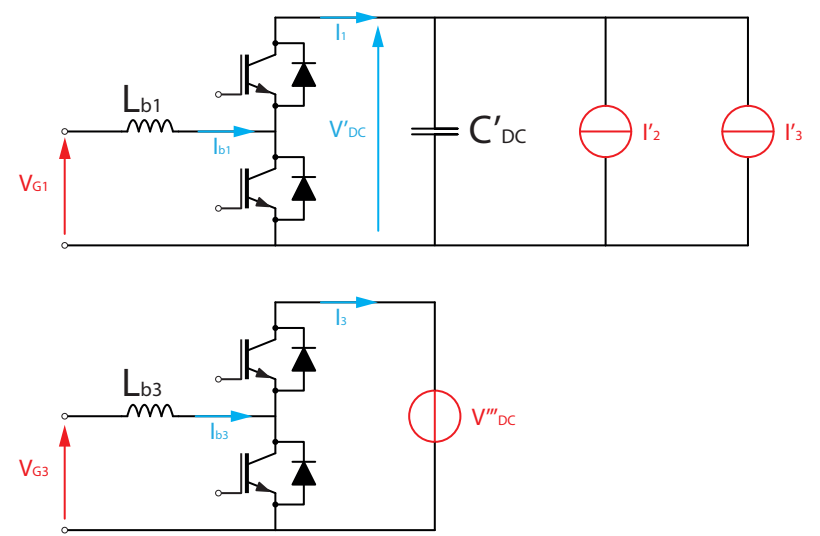

Fig. 4. Simplified model of the converter assuming unity transfer function of the resonant stage

referred to the third winding. The average model of two subcircuits from Fig. 4 is represented in Fig. 5, where $G_{I_{1}}(s)(18)$, $G_{I_{3}}(s)$ (20) are the transfer functions of the boost inductors $L_{B_{1}}, L_{B_{3}}$ and $G_{U c}(s)$ (19) is the transfer function of the combined DC capacitors $C_{D C}$.

$$
\begin{aligned}
C_{D C}^{\prime} & =C_{D C_{1}}+\frac{n_{2}^{2}}{n_{1}^{2}} C_{D C_{2}}+\frac{n_{3}^{2}}{n_{1}^{2}} C_{D C_{3}} \\
I_{2}^{\prime} & =\frac{n_{2}}{n_{1}} I_{2} \\
I_{3}^{\prime} & =\frac{n_{3}}{n_{1}} I_{3} \\
V_{D C}^{\prime \prime \prime} & =\frac{n_{3}}{n_{1}} V_{D C}^{\prime} \\
G_{I_{1}}(s) & =\frac{1 / R_{l_{1}}}{1+s\left(L_{b_{1}} / R_{l_{1}}\right)}=\frac{K_{I_{1}}}{1+s T_{I_{1}}} \\
G_{U c}(s) & =\frac{1}{s C_{D C}^{\prime}}=\frac{1}{s T_{U}} \\
G_{I_{3}}(s) & =\frac{1 / R_{l_{3}}}{1+s\left(L_{b_{3}} / R_{l_{3}}\right)}=\frac{K_{I_{3}}}{1+s T_{I_{3}}}
\end{aligned}
$$

\section{B. Control system}

Rather simple control approach has been followed and the voltage on the port 2 is regulated through two cascaded PI loops. The inner loop controls the inductor current $I_{B_{1}}$ with the PI regulator $G_{R I_{1}}$ while the outer loop controls the voltage $V_{D C_{2}}$ with the PI regulator $G_{R U}$. The current in the port 3 is regulated with a single PI loop and the regulator $G_{R I_{3}}$. Please note that in case of a connection of energy storage (e.g. battery, ultra-capacitors) there would be a need for outer loop for the purpose of the energy management. This is however not the scope of this paper and the used control structure is presented in Fig. 6, with the parameters of the regulators provided in the Table VI. These values are obtained according to description provided in the Appendix.

The control structure shown in Fig. 6 does not include all implementation details associated with the changes of the modes of operation. To avoid large inrush currents during the 


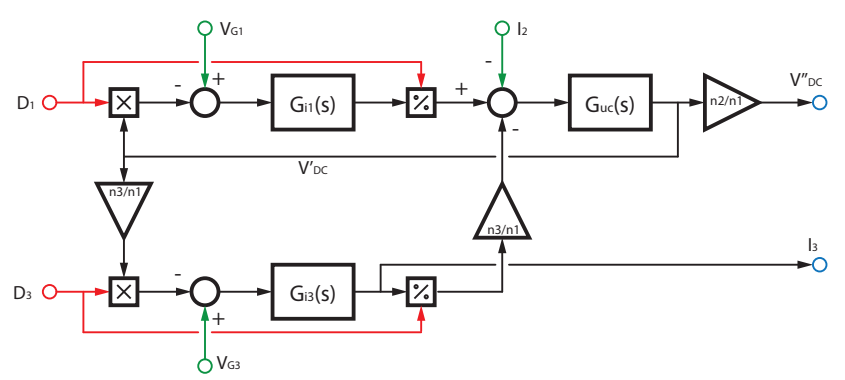

Fig. 5. Average model of the circuit from Fig. 4

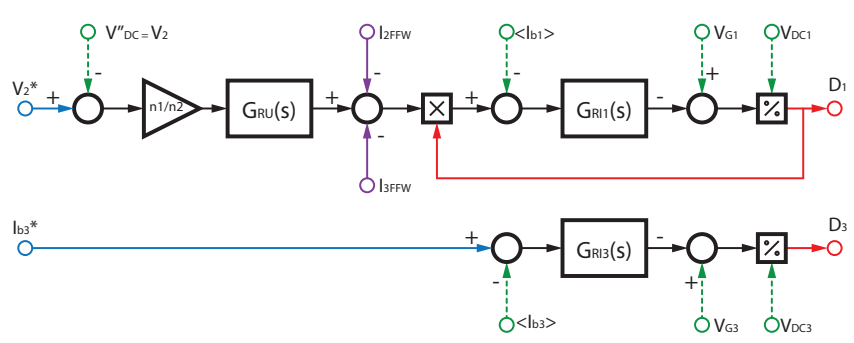

Fig. 6. Control structure used in conjunction with the model from Fig. 5

TABLE VI

PI REGULATOR'S PARAMETERS

\begin{tabular}{|l|l|l|l|}
\hline$T_{n_{I 1}}$ & 0.8 & $T_{i_{I 1}}$ & 0.012 \\
\hline$T_{n_{u c}}$ & 0.0032 & $T_{i_{u c}}$ & 0.0241 \\
\hline$T_{n_{I 3}}$ & 0.1 & $T_{i_{I 3}}$ & 0.018 \\
\hline
\end{tabular}

startup of $S_{1}, S_{2}$ or $S_{3}$, the duty cycle is ramp-up to final $50 \%$. During this transition sequence, the ZVS is not necessarily achieved.

\section{Simulation Results}

To verify design of the distributed resonant tank and the performance of the control scheme in the different modes of operation, different simulations have been performed. The closed-loop switched model has been implemented in PLECS as well as on the real-time power electronics hardware-in-theloop (HIL) simulator controlled with digital signal processor (DSP).

\section{A. PLECS Simulations}

Simulation results with several mode transitions are shown in Fig. 7 and 8. The unfiltered DC voltages of the resonant stage are shown in the upper plots in per unit values, while the RMS and average values of relevant currents, respectively, are shown in the bottom plots. Detailed description of the simulation scenarios is provided in the captions of Fig. 7 and 8. As it can be seen, the output voltage $V_{D C_{2}}$ has been effectively regulated to its rated value, in all modes of operation.

\section{B. HIL Simulations}

As a second step, the complete converter has been modeled and deployed on the Typhoon HIL 400 real-time power
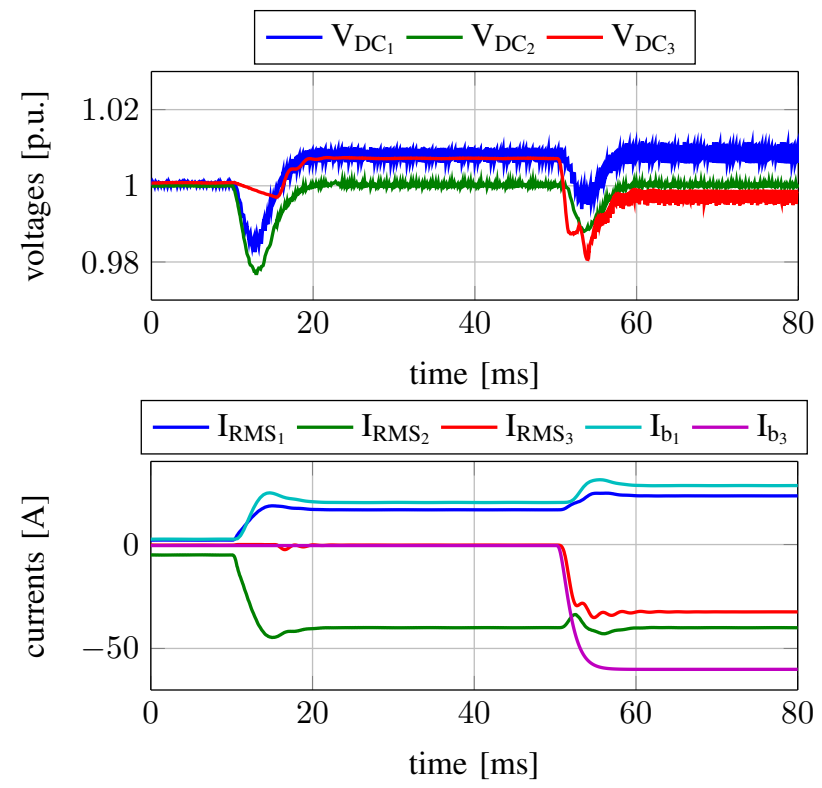

Fig. 7. PLECS simulations: At $\mathrm{t}=10 \mathrm{~ms}$, the load on the port 2 is increased from 0 to $-40 \mathrm{~A}$. The current in the boost $S_{4}$ tracks this current step resulting in an increase of $V_{D C_{1}}$. After the transient, the voltage $V_{D C_{2}}$ returns to its reference ( 1 p.u.). At $\mathrm{t}=50 \mathrm{~ms}$, the load on the port 3 is increased from 0 to $-60 \mathrm{~A}$ resulting in a decrease of $V_{D C_{3}}$. After the transient, $I_{R M S_{2}}$ remains the same and $I_{R M S_{1}}$ is slightly increased by its contribution in $I_{R M S_{3}}$. $V_{D C_{2}}$ is regulated back to 1 [p.u.]
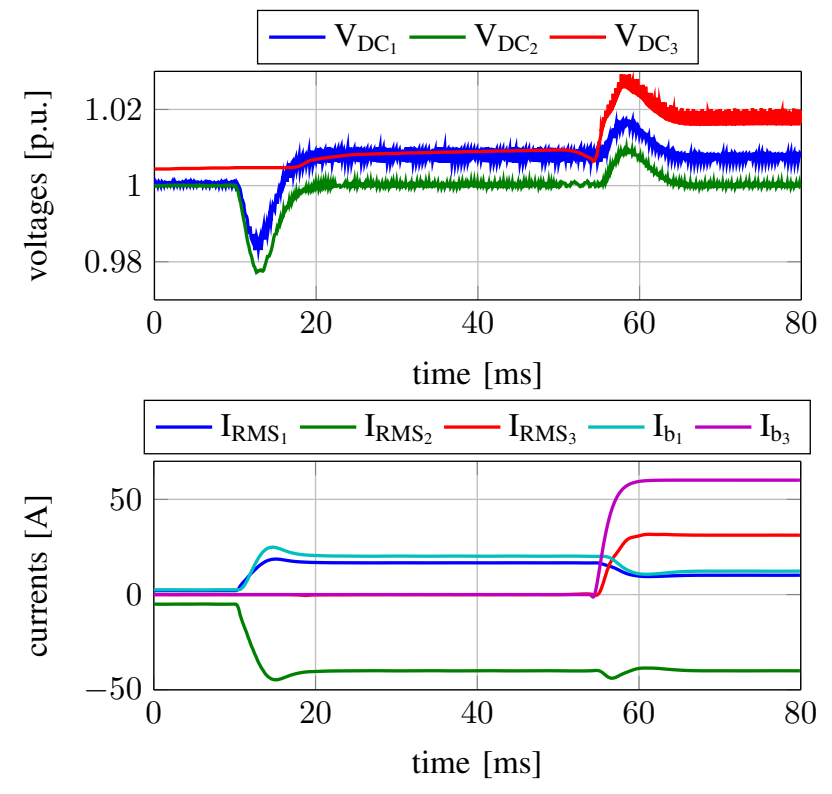

Fig. 8. PLECS simulations: At $\mathrm{t}=10 \mathrm{~ms}$, the load on the port 2 is increased from 0 to $-40 \mathrm{~A}$. $I_{B_{1}}$ follows this current step resulting in an increase of $V_{D C_{1}}$. Since there is no power exchange through the port $3, S_{5}$ is deactivated and $V_{D C_{3}}$ is following $V_{D C_{1}}$. At $\mathrm{t}=50 \mathrm{~ms}, S_{3}$ is turned on and starts with a modulation ramp. At $\mathrm{t}=55 \mathrm{~ms}$ the ramp is finished and the buck/boost stage $S_{5}$ of port 3 starts to regulate the current $I_{b_{3}}$ to $60 \mathrm{~A}$.

electronics simulator, while the control algorithm has been 


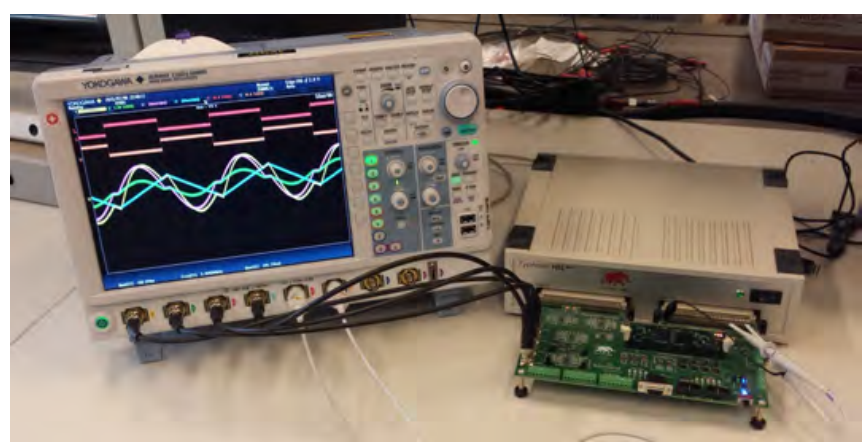

Fig. 9. HIL test setup with TI C2000 DSP

implemented on the TI TMS320F28335 DSP. This allows to carry out the simulations of numerous test cases and verify performances of the digital controller in the real-time. The complete test setup is shown in Fig. 9 (actual resonant current waveforms observed during testing are visible on the scope), while the captions of Fig. 10 and 11 provide description of the modes of the operation. Change of loading conditions on the port 3 has been tested and closed loop response of the control algorithm responsible for the main ports has been successfully verified.
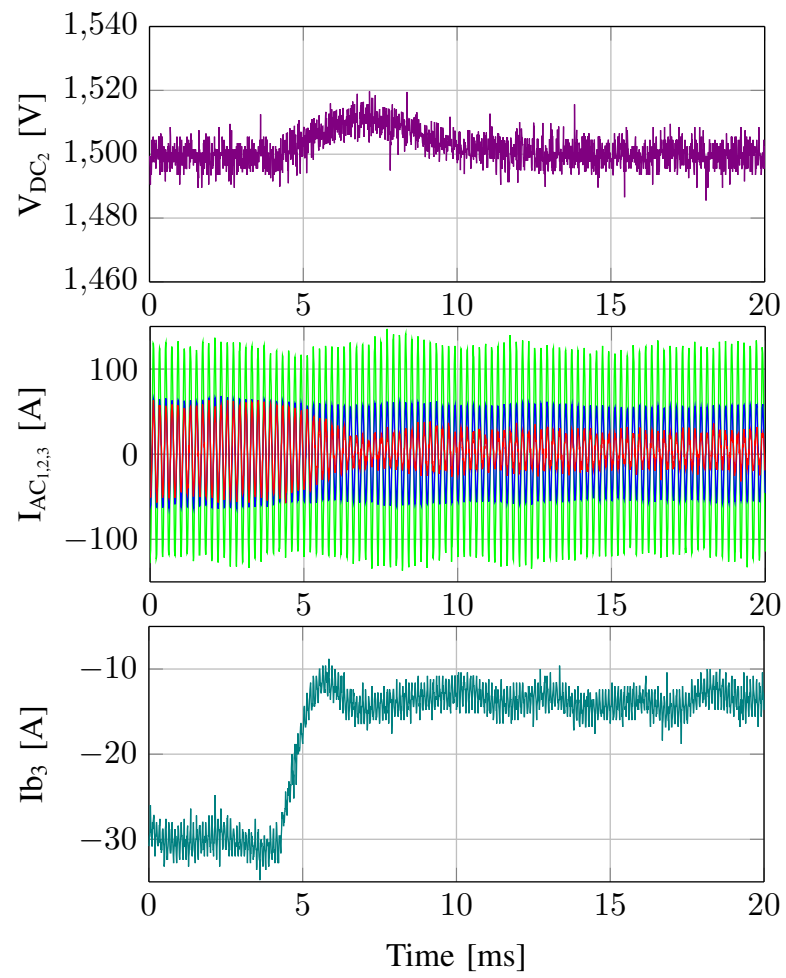

Fig. 10. HIL simulations: At $\mathrm{t}=4 \mathrm{~ms}$, the load on the port $3, I_{b_{3}}$ is decreased from -30 to $-15 \mathrm{~A}$. In response, the voltage on the port $2 V_{D C_{2}}$ is slightly increased, but immediately regulated by buck/boost stage $S_{4}$ of the port 1 . All three resonant tank currents are shown in the middle plot, from where their envelope values can be observed.

\section{Vi. CONCLUSIONS}

The topology of a bidirectional multiport resonant DC-DC converter has been presented in this paper. It is based on the combination of an open-loop operated multiport resonant converter with distributed resonant tank, and additional closedloop operated boost/buck stages on two ports. The operating modes of the converter have been described and suitable control scheme is presented. The inner resonant stage operates as DC transformer, and its main function is to provide galvanic isolation between different ports. Auxiliary third port has reduced ratings and is intended for connection of electrical energy storage elements, as support for the main ports.

Simulation results demonstrate operating principles, while further verification has been performed with digital controller deployed on the DSP and tested in the real-time with hardware-in-the-loop simulator. Topology, of the presented multiport converter, allow for further extensions for the higher operating voltages. Several converter stages from Fig. 1 can be stacked in input-series (port 1) output-parallel (port 2) fashion, with multiple ports 3 either left floating or tied together. In this way, connection to the higher DC voltages on the port 1 side is possible (insulation requirements for the MFT are naturally increased). This, together with the hardware design is the subject of the ongoing work, and will be reported separately.
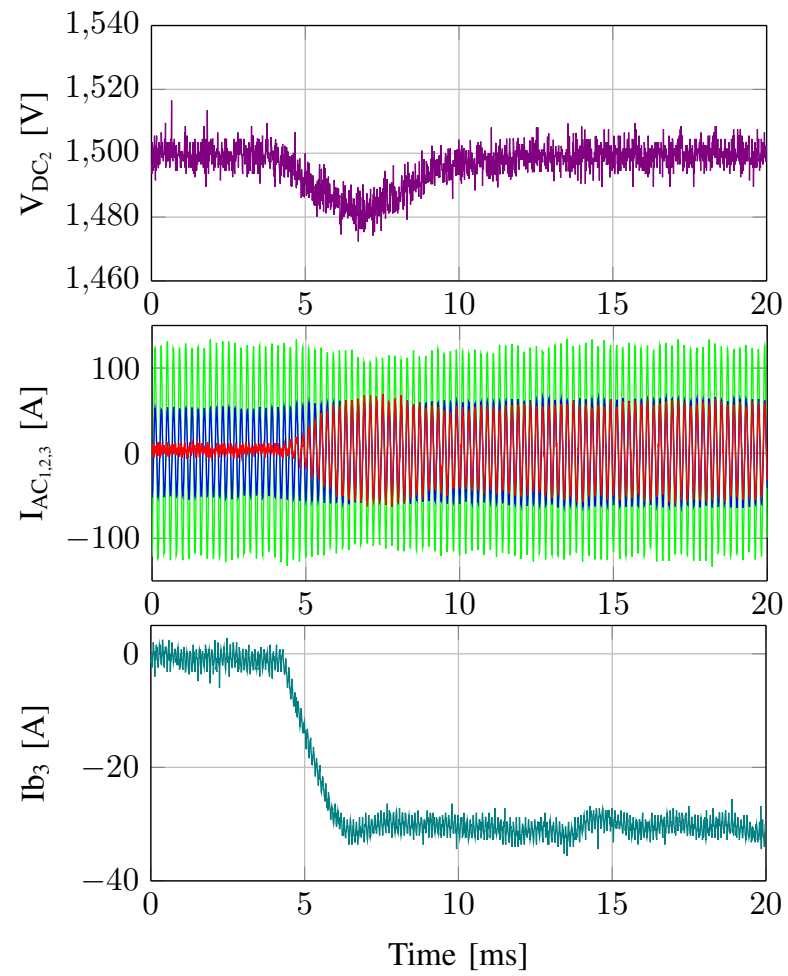

Fig. 11. HIL simulations: At $\mathrm{t}=4 \mathrm{~ms}$, the load on the port $3, I_{b_{3}}$ is increased from 0 to $-30 \mathrm{~A}$. In response, the voltage on the port $2 V_{D C_{2}}$ is slightly decreased, but immediately regulated by the buck/boost stage $S_{4}$ of the port 1. All three resonant tank currents are shown in the middle plot, from where their envelope values can be observed. 


\section{ACKNOWLEDGEMENT}

This research project is part of the National Research Programme "Energy Turnaround" (NRP70) of the Swiss National Science Foundation (SNSF). Further information on the National Research Programme can be found at www.nrp70.ch.

\section{APPENDIX}

The parameters of the regulators $G_{R I_{1}}$ and $G_{R I_{3}}$ are determined according to the principle of the symmetric optimum while the regulator $G_{R U}$ is tuned according to the principle of the magnitude optimum.

$$
\begin{aligned}
T_{P E_{1}} & =0.5 T_{\text {sample }}+T_{\text {sw } w_{\text {boost } 1}} \\
T_{n_{I 1}} & =T_{I_{1}} \\
T_{i_{I 1}} & =2 K_{I_{1}} T_{P E_{1}} \\
T_{C L} & =0.5 T_{\text {sample }}+2 T_{P E_{1}} \\
T_{n_{U}} & =4 T_{C L} \\
T_{i_{U}} & =8 T_{C L}^{2} / T_{U} \\
T_{P E_{3}} & =0.5 T_{\text {sample }}+T_{\text {sw }} \\
T_{n_{I 3}} & =T_{I_{3}} \\
T_{i_{I 3}} & =2 K_{I_{3}} T_{P E_{3}}
\end{aligned}
$$

Please note that for the simulation studies, the sampling time $T_{\text {sample }}$ is equal to the switching period of the resonant stage $T_{s w}$. Resulting parameters of the regulators are given in Table VI.

\section{REFERENCES}

[1] H. Tao, A. Kotsopoulos, J. L. Duarte, and M. A. M. Hendrix, "Family of multiport bidirectional dc-dc converters," IEE Proceedings - Electric Power Applications, vol. 153, no. 3, pp. 451-458, 2006.

[2] H. Tao, A. Kotsopoulos, J. L. Duarte, and M. A. M. Hendrix, "Triple-half-bridge bidirectional converter controlled by phase shift and pwm," in Proceedings of the 21st Annual IEEE Applied Power Electronics Conference and Exposition - APEC, 2006, pp. 1256-1262.
[3] H. Tao, A. Kotsopoulos, J. L. Duarte, and M. A. M. Hendrix, "Transformer-coupled multiport zvs bidirectional dc/dc converter with wide input range," Power Electronics, IEEE Transactions on, vol. 23, no. 2, pp. 771-781, 2008.

[4] H. Tao, A. Kotsopoulos, J. L. Duarte, and M. A. M. Hendrix, "A soft-switched three-port bidirectional converter for fuel cell and supercapacitor applications," in Proceedings of the 36th Annual IEEE Power Electronics Specialists Conference - PESC, 2005, pp. 2487-2493.

[5] Z. Pavlovic, J. A. Oliver, P. Alou, O. Garcia, and J. A. Cobos, "Bidirectional multiple port dc/dc transformer based on a series resonant converter," in 28th Annual IEEE Applied Power Electronics Conference and Exposition - APEC, 2013, pp. 1075-1082.

[6] H. Krishnaswami and N. Mohan, "Constant switching frequency series resonant three-port bi-directional dcdc converter," in 39th Annual IEEE Power Electronics Specialists Conference - PESC, 2008, pp. 1640-1645.

[7] Y.-K. Tran, D. Dujic, and P. Barrade, "Multiport Resonant DC-DC Converter," in Proceedings of the 41st Annual Conference of the IEEE Industrial Electronics Society - IECON, 2015, pp. 3839-3844.

[8] D. Dujic, G. Steinke, E. Bianda, S. Lewdeni-Schmid, C. Zhao, J. K. Steinke, and F. Canales, "Soft switching characterization of a $6.5 \mathrm{kV}$ IGBT for high power LLC resonant DC-DC converter," in Proceedings of International Power Conversion and Intelligent Motion Conference - PCIM, 2012, pp. 625-631.

[9] I.-O. Lee and G.-W. Moon, "The k-q analysis for an llc series resonant converter," Power Electronics, IEEE Transactions on, vol. 29, no. 1, pp. 13-16, 2014.

[10] R.-L. Lin and C.-W. Lin, "Design criteria for resonant tank of llc dc-dc resonant converter," in Proceedings of the 36th Annual Conference of IEEE Industrial Electronics Society - IECON, 2010, pp. 427-432. 